\title{
Originales
}

\section{Utilización de antibióticos en el tratamiento de infecciones por cocos grampositivos multirresistentes en pacientes críticos}

\author{
O. RODRÍGUEZ COLOMO ${ }^{a}$, F. ÁLVAREZ LERMA ${ }^{b}$, B. ÁLVAREZ SÁNCHEZ ${ }^{c}$, R. OLTRA CHORDÁa,

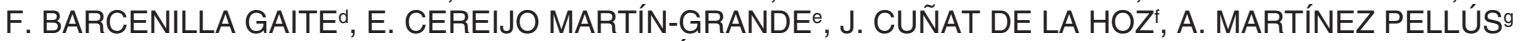 \\ $Y$ «GRUPO DE ESTUDIO DE USO DE ANTIBIÓTICOS ACTIVOS FRENTE A COCOS GRAM POSITIVOS \\ MULTIRRESISTENTES EN PACIENTES CRÍTICOS»*
}

\begin{abstract}
aServicio de Medicina Intensiva. Hospital Clínico Universitario. Valencia. España.

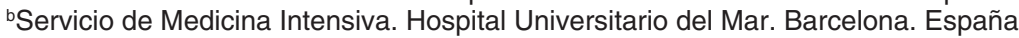
cServicio de Medicina Intensiva. Hospital General Universitario. Alicante. España.

'Servicio de Medicina Intensiva. Hospital Arnau de Vilanova. Lleida. España.

eServicio de Medicina Intensiva. Hospital de La Princesa. Madrid. España.

'Servicio de Medicina Intensiva. Hospital General La Fe. Valencia. España.

9Servicio de Medicina Intensiva. Hospital Virgen de la Arrixaca. Murcia. España.
\end{abstract}

Introducción. Este estudio se ha diseñado para conocer el uso de antibióticos (ATB) específicos frente a cocos grampositivos multirresistentes en Cuidados Intensivos.

Diseño y pacientes. Estudio observacional, prospectivo y multicéntrico. Se incluyeron como casos las indicaciones de vancomicina (VAN), teicoplanina (TPN), quimipristina/dalfopristina (Q/D) y linezolid (LZD).

Resultados. Se analizaron 826 indicaciones (VAN 52,1\%; TPN 36,6\%; LZD 11,6\% y Q/D 0\%), $793(96 \%)$ como tratamiento y $33(4 \%)$ como profilaxis en 818 pacientes. El 55,9\% de las infecciones se presentaron con sepsis grave o shock séptico. Las más frecuentes fueron neumonías y bacteriemias relacionadas con catéter, y el $48,3 \%$ fueron adquiridas en la Unidad de Cuidados Intensivos (UCI). LZD fue más utilizado en neumonías relacionadas con ventilación mecáni-

*Al final del trabajo se indican los profesionales que forman el «Grupo de Estudio de uso de Antibióticos Activos

frente a Cocos Gram Positivos Multirresistentes en Pacientes Críticos».

Correspondencia: Dr. O. Rodríguez Colomo.

Servicio de Medicina Intensiva.

Hospital Clínico Universitario de Valencia.

C/ Blasco Ibáñez, 17.

46010 Valencia. España.

Correo electrónico: rodríguez_osc@gva.es

Manuscrito aceptado el 6-XI-2007. ca $(p=0,001)$, VAN en infecciones del sistema nervioso central $(p=0,01)$ y en bacteriemias relacionadas con catéter $(p=0,001)$ y TPN en neumonías comunitarias $(p=0,01)$ y en bacteriemias relacionadas con catéter $(p=0,001)$. El $65,8 \%$ de los tratamientos fueron empíricos. Staphylococcus aureus resistente a meticilina fue el más aislado en el grupo de pacientes tratados con LZD. El tratamiento inicial se modificó en 224 ocasiones, circunstancia más frecuente en tratados con VAN. El motivo de esta modificación fue por fracaso clínico en 59 ocasiones, siendo más frecuente en el grupo tratado con TPN y menos en el tratado con LZD, que además fue el fármaco más utilizado en los tratamientos de rescate $(43 / 69,62,3 \%)$. Se han identificado efectos adversos probablemente relacionados con los ATB en $36 / 826(4,4 \%)$ casos.

Conclusiones. Las indicaciones más frecuentes fueron para tratar infecciones adquiridas en la UCI. VAN fue el fármaco más utilizado. El tratamiento con LZD se modificó en menos ocasiones por fracaso clínico y fue el más empleado como tratamiento de rescate. El conjunto de la información muestra un empleo adecuado de estos ATB en un importante porcentaje de pacientes críticos.

PALABRAS CLAVE: cocos grampositivos multirresistentes, paciente crítico, tratamiento antibiótico. 


\section{USE OF ANTIBIOTICS FOR THE TREATMENT OF MULTIRESISTANT GRAM POSITIVE COCCI INFECTIONS IN CRITICAL PATIENTS}

Introduction. This study has been designed to know the use of these antibiotics (ATB) in Intensive Care Units (ICUs).

Design and patients. A multicentric, prospective, observational study was conducted. Included as cases were the indications of vancomycin (VAN), teicoplanin (TPN), quinupristin/dalfopristin (Q/D) and linezolid (LZD).

Results. A total of 826 indications (VAN $52.1 \%$, TPN $36.6 \%$, LZD $11.6 \%$ and Q/D $0 \%$ ) were analyzed, $793(96 \%)$ as treatment and $33(4 \%)$ as prophylaxis in 818 patients. Serious sepsis or septic should occurred in $55.9 \%$ of the patients treated. The most common infections were pneumonia and catheter-related bacteremia, $48.3 \%$ of all the infections being acquired in ICUs. LZD was used mostly in mechanical-ventilation related pneumonia $(p=0.001)$, VAN in community Central Nervous System infections $(p=0.01)$ and in catheter-related bacteremia $(p=0.001)$, TPN in community pneumonia $(p=0.01)$ and in catheter-related bacteremia $(p=0.001)$. Treatments were empirical in $65.8 \%$ and diagnosis of gram positive cocci was confirmed in $48.3 \%$ of them. Staphylococcus aureus was isolated more in the LZD-treated group. Initial treatment was modified 224 times, this occurring more frequently in the VAN-treated group. The reason for this change was clinical failure 59 times, this being more frequent in the TPN group and less frequent in the patients treated with LZD, which was the drug used the most in rescue therapies $(43 / 69,62.3 \%)$. Adverse events, probably related to the ATB, were identified in 36/826 (4.4\%) cases.

Conclusions. The most common use of this ATB was to treat ICU-acquired infections. VAN was the most frequently used drug. Treatments with LZD were modified less frequently for clinical failure and this drug was the one most used in rescue therapies. This information indicates an appropriate use of these ATB in an important percentage of critically patients.

KEY WORDS: multiresistant gram positive cocci, critical paients, antibiotics treatment.

\section{INTRODUCCIÓN}

Las infecciones producidas por cocos grampositivos multirresistentes (CGP-MR) constituyen un grave problema sanitario, y su incidencia ha aumentado especialmente en el ámbito del paciente crítico. Numerosos factores han influido en ello, algunos relacionados con los propios pacientes (mayor edad, gravedad y presencia de comorbilidades), otros asociados con los tratamientos instaurados (antibióticos de amplio espectro, procedimientos invasivos) y fi- nalmente los debidos a la infraestructura de los centros hospitalarios (habitaciones abiertas, personal sanitario insuficiente o en periodo de formación). Hay un amplio consenso en que estas condiciones son propicias para la selección y transmisión de CGP-MR.

La epidemiología de las infecciones por CGP-MR ha variado notablemente a lo largo del tiempo. Los microorganismos grampositivos aislados preferentemente en la década de los cincuenta del siglo pasado perdieron protagonismo en las siguientes décadas en favor de los gramnegativos. En los últimos 20 años esta tendencia se ha invertido y las infecciones por grampositivos han sufrido un notable incremento ${ }^{1-8}$ tras la aparición de CGP-MR ${ }^{9-11}$. En España, y según datos del Estudio Nacional de Vigilancia de Infección Nosocomial (ENVIN) ${ }^{4-6}$, la presencia de Staphylococcus aureus resistente a meticilina (SARM) se ha incrementado paulatinamente en los pacientes ingresados en Servicios o Unidades de Cuidados Intensivos (UCI) entre los años 1994-2006, llegando en el último año de este periodo al $42,3 \%$ de todos los aislados de $S$. aureus. Lo mismo sucede con Staphylococcus coagulasa negativo ( $\mathrm{SCN}$ ) con tasas de resistencia a meticilina superiores al $80 \%$ en los últimos años. Por el contrario, la resistencia a glucopéptidos de ambas bacterias fue prácticamente nula, al igual que frente a linezolid. Paralelamente la presencia de Enterococcus spp. resistentes a glucopéptidos en España en infecciones adquiridas en UCI continúan siendo bajas, inferiores al $1 \%$, a diferencia de los datos del National Nosocomial Infection Surveillance System $^{8}$ (NNIS) del año 2003 en donde el porcentaje de Enterococcus spp. resistentes a vancomicina en la UCI era del $28,5 \%$

En consonancia con esta situación epidemiológica la utilización de antibióticos (ATB) específicos frente a CGP-MR se ha incrementado paulatinamente. Actualmente estos ATB, en conjunto, son los que más se utilizan en el ámbito del paciente crítico para el tratamiento de las infecciones nosocomiales adquiridas en la UCI, así como en los tratamientos dirigidos de estas infecciones ${ }^{12-14}$. Desde la década de los cincuenta con la aparición de vancomicina (VAN), y teicoplanina (TPN) en los noventa, no habían surgido nuevos fármacos activos frente a CGP-MR hasta la incorporación de quinupristina/dalfopristina (Q/D) y linezolid (LZD) ${ }^{15-19}$, y más recientemente daptomicina y tigeciclina. Actualmente hay muchos otros en fase de investigación.

Este estudio se ha diseñado para conocer los motivos y formas de empleo de los ATB específicos para el tratamiento de CGP-MR en áreas de pacientes críticos.

\section{PACIENTES Y MÉTODOS}

Se ha realizado un estudio observacional, prospectivo y multicéntrico, en el que han participado $38 \mathrm{UCI}$ ampliamente distribuidas por la geografía española, predominando la participación de Cataluña, Andalucía y la Comunidad Valenciana, que en conjunto suman el 52,6\% de los centros participantes. Los datos 
se han recogido entre enero de 2003 y abril de 2004. Se han incluido como caso, y de forma consecutiva, todos los pacientes ingresados en la UCI en los que se ha prescrito VAN, TPN, Q/D o LZD (daptomicina y tigeciclina aún no estaban comercializadas), en monoterapia o en combinación con otros antimicrobianos, en infecciones por CGP-MR demostradas o sospechadas en cualquier localización, así como en profilaxis. Se ha definido como CGP-MR el Staphylococcus aureus y el SCN resistentes a la meticilina, así como los que también son resistentes a VAN, y el enterococo resistente a VAN. El seguimiento de los pacientes se realizó hasta la finalización del tratamiento valorando la respuesta clínica y microbiológica y la tolerabilidad.

En un cuaderno de recogida de datos se registraron variables demográficas, antecedentes patológicos, diagnóstico de ingreso en UCI y los factores de riesgo de adquirir una infección. También se registró la localización y el tipo de infección, su forma de presentación, las bacterias implicadas y su sensibilidad frente a los ATB estudiados, así como la repercusión en las pautas terapéuticas, la forma de utilización de los ATB y las características del tratamiento, incluidas las dosis utilizadas, además de la tolerancia y aparición de resistencias. Entre los factores de riesgo de infección se incluyó la inserción de catéteres intravasculares, la ventilación mecánica, la colocación de sonda urinaria y el empleo de técnicas de depuración extrarrenal. La gravedad al ingreso se calculó mediante la escala APACHE II, y la forma de presentación de las infecciones se ha clasificado siguiendo los criterios de Bone $^{20}$ en el síndrome de respuesta inflamatoria sistémica (SRIS), la sepsis grave y el shock séptico.

Se catalogaron como corticoterapia aquellos casos en los que el paciente recibía más de $40 \mathrm{mg} /$ día de prednisona o su equivalente, durante por lo menos 15 días.

Las infecciones se clasificaron como comunitarias y hospitalarias, diferenciándose estas últimas entre las adquiridas en la UCI y fuera de ella. Se han considerado infecciones hospitalarias las diagnosticadas pasadas las 48 horas del ingreso, y adquiridas en la UCI las que se diagnosticaron después de 48 horas de estancia en la misma. Las indicaciones de los ATB se clasificaron en profilaxis y tratamiento, distinguiendo estas últimas entre tratamiento empírico o específico, en función del conocimiento previo de los agentes causales, así como de su sensibilidad frente a los ATB estudiados. Así mismo, se registró si el tratamiento se administró en monoterapia o en terapia combinada, las dosis utilizadas y la antibioterapia concomitante. Por otro lado, se reflejó si hubo modificaciones del tratamiento ATB inicial y sus causas, así como el tratamiento de rescate y su efectividad clínica y microbiológica. Se ha considerado tratamiento de rescate al realizado con ATB indicados en segunda intención, prescritos por cualquier motivo y después de por lo menos 48 horas de seguir el tratamiento inicial. Se excluyen de esta definición aquellos que se indican para ajustar, desescalar o realizar una terapia secuencial. Las enfermedades de base se han clasificado en médicas, quirúrgicas, co- ronarias y traumáticas. Los diagnósticos de neumonía se han realizado de acuerdo con las normas definidas por el Centers for Disease Control and Prevention.

\section{Análisis estadístico}

Se realizó un análisis descriptivo presentando las variables cualitativas en forma de porcentaje y las cuantitativas como medias en caso de presentar una distribución normal, o como mediana y rango en caso contrario. Las comparaciones entre los pacientes tratados con diferentes ATB se han realizado utilizando la Chi cuadrado para variables cualitativas mediante la prueba exacta de Fisher para tablas $2 \times 2$. Para variables cuantitativas se utilizaron pruebas ANOVA en caso de poder ser asumida la normalidad, o la prueba de Kruskal-Wallis en caso contrario. La hipótesis de normalidad fue comprobada mediante procedimientos gráficos y el test de Kolmogorov-Smirnov. En todos los contrastes se consideraron pruebas bilaterales con un nivel de significación de 0,05 . Para todos los análisis se utilizó el programa SPSS ${ }^{\circledR 13}$.

\section{RESULTADOS}

Se incluyeron 818 pacientes que recibieron alguno de los ATB estudiados en 826 ocasiones. La media de inclusión fue de 22 episodios por centro (mínimo 5, máximo 80). En 5 pacientes se administraron 13 ATB en periodos no consecutivos. En los 826 episodios estudiados VAN fue indicada en $430(52,1 \%)$ ocasiones, TPN en $300(36,6 \%)$ casos y LZD en 96 (11,6\%). No se observó ninguna prescripción de Q/D.

Las características de los pacientes incluidos y sus variables demográficas se muestran en la tabla 1. Se han observado diferencias significativas entre los grupos dependiendo del ATB utilizado, de la gravedad, de la estancia en la UCI, de la patología de base y de la mortalidad. Los antecedentes patológicos y los factores de riesgo de infección se incluyen en la tabla 2 , donde se comparan en función de cada uno de los tratamientos recibidos. Entre los pacientes tratados con LZD predominaron de forma significativa, con respecto a los pacientes tratados con VAN, la diabetes mellitus, la insuficiencia renal crónica, el trasplante de órganos y el empleo de corticoides. Por otro lado, los pacientes tratados con TPN recibieron más tratamiento inmunosupresor que aquellos con VAN $(\mathrm{p}=0,005)$. Así mismo, en los pacientes que siguieron tratamiento con LZD se emplearon de forma diferencial más catéteres en la arteria pulmonar y más técnicas de depuración extrarrenal que en los que se pautó VAN.

En 793 (96\%) casos el motivo de administrar los ATB en estudio ha sido el tratamiento de infecciones y en $33(4 \%)$ ocasiones la profilaxis. Las infecciones tratadas han sido nosocomiales adquiridas en la UCI (intra-UCI) en $483(60,9 \%)$ ocasiones, nosocomiales adquiridas fuera de la UCI (extra-UCI) en $174(21,9 \%)$ casos y comunitarias en $136(17,2 \%)$. Un tercio de las indicaciones profilácticas se hicieron para cirugía cardiaca (11/33) y sólo en un caso para cirugía or- 
TABLA 1. Variables demográficas y características de los pacientes tratados con antibióticos activos frente a cocos grampositivos multirresistentes

\begin{tabular}{|c|c|c|c|c|c|}
\hline & $\begin{array}{c}\text { Total } \\
\mathrm{n}=818\end{array}$ & $\begin{array}{c}\text { VAN } \\
\mathrm{n}=426\end{array}$ & $\begin{array}{c}\text { TPN } \\
\mathrm{n}=299\end{array}$ & $\begin{array}{c}\text { LZD } \\
\mathrm{n}=93\end{array}$ & $\mathrm{p}$ \\
\hline Edad (años), mediana (mín-máx) & $62(13-87)$ & $61(13-87)$ & $62(15-87)$ & $66(19-79)$ & NS \\
\hline Sexo masculino $(\%)$ & $556(68,0)$ & $279(65,5)$ & $210(70,2)$ & $67(72,0)$ & NS \\
\hline APACHE II, mediana (mín-máx) & $17(0-44)$ & $16(0-44)$ & $18(3-39)$ & $18(3-39)$ & $0,003^{\mathrm{A}}$ \\
\hline Estancia en UCI (días), mediana & 19 & 18 & 18 & 21 & $0,012^{\mathrm{B}}$ \\
\hline Exitus $(\%)$ & $277(33,9)$ & $111(26,1)^{*}$ & $119(39,8)^{*}$ & $47(50,5) *$ & $<0,001^{\mathrm{C}}$ \\
\hline \multicolumn{6}{|l|}{ Tipo de paciente $\mathrm{n}(\%)$} \\
\hline Pacientes médicos & $459(56,1)$ & $249(58,5)$ & $163(54,5)$ & $50(50,5)$ & \\
\hline Pacientes quirúrgicos & $245(30,0)$ & $115(27,0) *$ & $91(30,4)$ & $39(41,9)^{*}$ & $0,039^{\mathrm{D}}$ \\
\hline Pacientes traumáticos & $97(11,9)$ & $55(12,9)$ & $35(11,7)$ & $7(7,5)$ & \\
\hline Pacientes coronarios & $17(2,1)$ & $7(1,6)$ & $10(3,3)$ & $0(0,0)$ & \\
\hline
\end{tabular}

Residuos tipificados corregidos con valor absoluto > 1,96; LZD: linezolid; TPN: teicoplanina; VAN: vancomicina

A: VAN frente a TPN y LZD; ${ }^{\text {B: }}$ LZD frente a VAN y TPN; ${ }^{\text {C: }}$ VAN frente a TPN y LZD; ${ }^{\text {D: }}$ LZD frente a VAN.

Nota: en los 5 pacientes con más de un tratamiento se ha seleccionado sólo el primero para no repetir enfermos.

topédica. Las infecciones se presentaron como sepsis grave o shock séptico en $443(55,9 \%)$ casos. En la tabla 3 se incluye la distribución de los ATB estudiados para cada una de las indicaciones, el tipo de infección y la forma de presentación de las mismas. Destaca un mayor número de pacientes con sepsis grave o shock séptico entre los que han recibido LZD o TPN.

La localización de las infecciones tratadas con los ATB en estudio ha sido neumonía en $273(34,4 \%)$ casos, seguida de bacteriemia relacionada con catéter (BRC) en $212(26,7 \%)$ ocasiones, siendo menos frecuentes las infecciones de piel y partes blandas con 40 $(5,9 \%)$ casos, las infecciones del sistema nervioso central (SNC) con 53 episodios $(6,7 \%)$ y las infecciones quirúrgicas de órgano o espacio con 40 casos $(5,0 \%)$. En 47 ocasiones se administraron en síndromes febriles sin foco conocido de infección. En la ta- bla 4 se incluye la utilización de cada uno de los ATB controlados dependiendo del lugar de adquisición de la infección y de su localización. Ha predominado de forma significativa la utilización de LZD en neumonías relacionadas con ventilación mecánica $(\mathrm{NVM})(\mathrm{p}=0,001)$, el empleo de VAN en infecciones del SNC comunitarias $(\mathrm{p}=0,01)$ y en BRC adquiridas en la UCI ( $p=0,001)$, mientras que TPN se ha utilizado en neumonías adquiridas en la comunidad $(\mathrm{p}=$ $0,01)$ y en BRC adquiridas en la UCI $(p=0,001)$.

La forma de administrar los ATB se incluye en la tabla 5. De las 793 indicaciones para tratamiento, en 522 la prescripción se hizo en forma empírica $(65,8 \%)$. El diagnóstico de infección por CGP se confirmó posteriormente en el $48,3 \%$ de los casos (252/522). En 10 casos no se solicitó cultivo previo a la administración del ATB; en 132 (16\%) los fárma-

TABLA 2. Antecedentes patológicos y factores de riesgo de los pacientes en función del antibiótico utilizado

\begin{tabular}{|c|c|c|c|c|c|}
\hline & $\begin{array}{c}\text { Total } \\
\mathrm{n}=818\end{array}$ & $\begin{array}{c}\text { VAN } \\
\mathrm{n}=426\end{array}$ & $\begin{array}{c}\text { TPN } \\
\mathrm{n}=299\end{array}$ & $\begin{array}{c}\text { LZD } \\
\mathrm{n}=93\end{array}$ & $\mathrm{p}$ \\
\hline \multicolumn{6}{|l|}{ Antecedentes patológicos } \\
\hline Diabetes mellitus & $179(21,9)$ & $79(18,5)^{*}$ & $71(23,7)$ & $29(31,2)^{*}$ & 0,018 \\
\hline Insuficiencia renal crónica & $69(8,4)$ & $24(5,6)^{*}$ & $28(9,4)$ & $17(18,3)^{*}$ & $<0,001$ \\
\hline EPOC & $142(17,4)$ & $77(18,1)$ & $48(16,1)$ & $17(18,3)$ & 0,755 \\
\hline Insuficiencia cardiaca severa & $55(6,7)$ & $23(5,4)$ & $25(8,4)$ & $7(7,5)$ & 0,277 \\
\hline Hepatopatía crónica & $51(6,2)$ & $24(5,6)$ & $18(6,0)$ & $9(9,7)$ & 0,338 \\
\hline Alcoholismo & $68(8,3)$ & $34(8,0)$ & $30(10,0)$ & $4(4,3)$ & 0,203 \\
\hline Neoplasia sólida & $88(10,8)$ & $45(10,6)$ & $37(12,4)$ & $6(6,5)$ & 0,269 \\
\hline Neoplasia hematológica & $44(5,4)$ & $22(5,2)$ & $19(6,4)$ & $3(3,2)$ & 0,486 \\
\hline Inmunodeficiencia & $49(6,0)$ & $22(5,2)$ & $20(6,7)$ & $7(7,5)$ & 0,558 \\
\hline Trasplante de órganos & $29(3,5)$ & $8(1,9)^{*}$ & $14(4,7)$ & $7(7,5)^{*}$ & 0,012 \\
\hline Quemaduras & $6(0,7)$ & $3(0,7)$ & $3(1,0)$ & $0(0,0)$ & 0,609 \\
\hline Corticoterapia & $67(8,2)$ & $23(5,4)^{*}$ & $30(10,0)$ & $14(15,1)^{*}$ & 0,003 \\
\hline Tratamiento inmunosupresor & $77(9,4)$ & $27(6,3)^{*}$ & $40(13,4)^{*}$ & $10(10,8)$ & 0,005 \\
\hline \multicolumn{6}{|l|}{ Factores de riesgo } \\
\hline Ventilación mecánica & $605(74,0)$ & $311(73,0)$ & $227(75,9)$ & $67(72,0)$ & 0,614 \\
\hline Sonda urinaria & $771(94,3)$ & $394(92,5)$ & $288(96,3)$ & $89(95,7)$ & 0,075 \\
\hline Catéter venoso central & $757(92,5)$ & $387(90,8)$ & $284(95,0)$ & $86(92,5)$ & 0,113 \\
\hline Catéter arterial & $503(61,5)$ & $259(60,8) *$ & $175(58,5)^{*}$ & $69(74,2)^{*}$ & $0,023^{\mathrm{A}}$ \\
\hline Catéter de la arteria pulmonar & $79(9,7)$ & $28(6,6)^{*}$ & $33(11,0)$ & $18(19,4) *$ & $<0,001$ \\
\hline Técnicas de depuración extrarrenal & $82(10,0)$ & $31(7,3)^{*}$ & $33(11,0)$ & $18(19,4) *$ & 0,002 \\
\hline
\end{tabular}

*Residuos tipificados corregidos con valor absoluto $>1,96$. 
TABLA 3. Distribución de los antibióticos estudiados dependiendo del motivo de la indicación, del tipo de infección y de su forma de presentación

\begin{tabular}{|c|c|c|c|c|c|}
\hline Indicaciones $\mathrm{n}(\%)$ & $\begin{array}{c}\text { Total } \\
\mathrm{n}=826\end{array}$ & $\begin{array}{c}\text { VAN } \\
\mathrm{n}=430\end{array}$ & $\begin{array}{c}\text { TPN } \\
\mathrm{n}=300\end{array}$ & $\begin{array}{c}\text { LZD } \\
\mathrm{n}=96\end{array}$ & $\mathrm{p}$ \\
\hline Tratamiento & $793(96)$ & $405(94,2)$ & $293(97,7)$ & $95(99)$ & \\
\hline Profilaxis & $33(4)$ & $25(6,8)$ & $7(2,3)$ & $1(1,0)$ & \\
\hline Tipo de infección, n (\%) & $\begin{array}{c}\text { Total } \\
\mathrm{n}=793\end{array}$ & $\begin{array}{c}\text { VAN } \\
\mathrm{n}=405\end{array}$ & $\begin{array}{c}\text { TPN } \\
\mathrm{n}=293\end{array}$ & $\begin{array}{c}\text { LZD } \\
\mathrm{n}=95\end{array}$ & $\mathrm{p}$ \\
\hline Comunitarias & $136(17,2)$ & $85(21,0)^{*}$ & $44(15,0)$ & $7(7,4)^{*}$ & 0,009 \\
\hline Hospitalarias extra-UCI & $174(21,9)$ & $77(19,0)$ & $71(24,2)$ & $26(27,4)$ & \\
\hline Hospitalarias intra-UCI & $483(60,9)$ & $243(60,0)$ & $178(60,8)$ & $62(65,3)$ & \\
\hline Forma de presentación, n (\%) & Total & VAN & TPN & LZN & $\mathrm{p}$ \\
\hline Sin sepsis & $67(8,4)$ & $34(8,4)$ & $25(8,5)$ & $8(8,4)$ & \\
\hline Sepsis leve & $283(35,7)$ & $171(42,2)$ & $85(29,0)$ & $27(28,4)$ & \\
\hline Sepsis grave & $209(26,4)$ & $103(25,4)$ & $81(27,6)$ & $25(26,3)$ & \\
\hline Shock séptico & $234(29,5)$ & $97(24,0)^{*}$ & $102(34,8)^{*}$ & $35(36,8)$ & 0,004 \\
\hline Sepsis grave/shock séptico & $443(55,9)$ & $200(49,4)^{*}$ & $183(62,5)^{*}$ & $60(63,2)$ & 0,003 \\
\hline
\end{tabular}

*Residuos tipificados corregidos con valor absoluto $>1,96$

LZD: linezolid; TPN: teicoplanina; VAN: vancomicina.

cos se administraron en monoterapia y en el resto en asociación con uno o más ATB. Las asociaciones más frecuentes fueron con imipenem-cilastatina en 138 casos, con amicacina en 110 , con cefepime en 106 , con piperacilina/tazobactam y con meropenem en 99 casos cada uno y en 90 casos con ciprofloxacino. Si la función renal era normal las dosis de VAN fueron de 1 g cada 12 horas y la duración media del tratamiento de 9,26 días; no hubo ningún caso con perfusión continua. Las tres primeras dosis de TPN fueron de $400 \mathrm{mg}$ cada 12 horas, seguidas de $400 \mathrm{mg}$ cada 24 horas. Sólo en 4 casos con insuficiencia renal crónica se administraron $200 \mathrm{mg}$ al día. En cuanto a las dosis de LZD fueron de $600 \mathrm{mg}$ cada 12 horas. Se

TABLA 4. Distribución de los antibióticos estudiados dependiendo del lugar de adquisición de las infecciones y de su localización

\begin{tabular}{|c|c|c|c|c|c|}
\hline & Total & VAN & TPN & LZD & $\mathrm{p}$ \\
\hline \multicolumn{6}{|l|}{ Infecciones comunitarias: $\mathrm{n}(\%)$} \\
\hline Neumonías & $52(38,2)$ & $27(31,8)^{*}$ & $24(54,5)^{*}$ & $1(14,3)$ & 0,010 \\
\hline Bacteriemias secundarias & $10(7,4)$ & $5(5,9)$ & $4(9,1)$ & $1(14,3)$ & \\
\hline Infecciones de partes blandas & $11(8,1)$ & $6(7,1)$ & $4(9,1)$ & $1(14,3)$ & \\
\hline Infecciones del SNC & $36(26,5)$ & $33(38,8)^{*}$ & $2(4,5)^{*}$ & $1(14,3)$ & 0,010 \\
\hline Síndrome febril tratado con ATB & $1(0,7)$ & $1(1,2)$ & $0(0,0)$ & $0(0,0)$ & \\
\hline Otras & $26(19,1)$ & $13(15,3)$ & $10(22,7)$ & $3(42,9)$ & \\
\hline Total infecciones comunitarias & $136(100)$ & $85(62,5)$ & $44(32,4)$ & $7(5,1)$ & \\
\hline \multicolumn{6}{|l|}{ Infecciones nosocomiales extra-UCI: n (\%) } \\
\hline Neumonías & $57(32,8)$ & $25(32,5)$ & $24(33,8)$ & $8(30,8)$ & \\
\hline Bacteriemias relacionadas con catéter & $38(21,8)$ & $12(15,6)$ & $23(32,4)$ & $3(11,5)$ & \\
\hline Bacteriemias secundarias & $15(8,6)$ & $7(9,1)$ & $4(5,6)$ & $4(15,4)$ & \\
\hline Infecciones de partes blandas & $12(6,9)$ & $6(7,8)$ & $2(2,8)$ & $4(15,4)$ & \\
\hline Infección quirúrgica de órgano o espacio & $23(13,2)$ & $11(14,3)$ & $8(11,3)$ & $4(15,4)$ & \\
\hline Infecciones del SNC & $4(2,3)$ & $4(5,2)$ & $0(0,0)$ & $0(0,0)$ & \\
\hline Síndrome febril tratado con ATB & $13(7,5)$ & $7(9,1)$ & $5(7,0)$ & $1(3,8)$ & \\
\hline Otras & $12(6,9)$ & $5(6,5)$ & $5(7,0)$ & $2(7,7)$ & \\
\hline Total nosocomiales extra-UCI & $174(100)$ & $77(44,3)$ & $71(40,8)$ & $26(14,9)$ & \\
\hline \multicolumn{6}{|l|}{ Infecciones nosocomiales intra-UCI: n (\%) } \\
\hline NVM & $164(34,0)$ & $71(29,2)^{*}$ & $62(34,8)$ & $31(50,0)^{*}$ & 0,001 \\
\hline Bacteriemias relacionadas con catéter & $174(36,0)$ & $93(38,3)^{*}$ & $69(38,8)^{*}$ & $12(19,4)^{*}$ & 0,001 \\
\hline Bacteriemias secundarias & $33(6,8)$ & $22(9,1)^{*}$ & $7(3,9)^{*}$ & $4(6,5)$ & 0,001 \\
\hline Infección urinaria relacionada con sonda & $8(1,7)$ & $5(2,1)$ & $1(0,6)$ & $2(3,2)$ & \\
\hline Infecciones de partes blandas & $17(3,5)$ & $8(3,3)$ & $5(2,8)$ & $4(6,5)$ & \\
\hline Infección quirúrgica de órgano o espacio & $17(3,5)$ & $4(1,6)^{*}$ & $12(6,7)^{*}$ & $1(1,6)$ & 0,001 \\
\hline Infecciones del SNC & $13(2,7)$ & $12(4,9)^{*}$ & $0(0,0)^{*}$ & $1(1,6)$ & 0,001 \\
\hline Síndrome febril tratado con ATB & $33(6,8)$ & $16(6,6)$ & $13(7,3)$ & $4(6,5)$ & \\
\hline Otras & $24(5,0)$ & $12(4,9)$ & $9(5,1)$ & $3(4,8)$ & \\
\hline Total nosocomiales intra-UCI & $483(100)$ & $243(50,3)$ & $178(36,9)$ & $62(12,8)$ & \\
\hline
\end{tabular}

*Residuos tipificados corregidos con valor absoluto $>1,96$ ATB: antibióticos; LZD: linezolid; NVM: neumonía relacionada con ventilación mecánica; SNC: sistema nervioso central; TPN: teicoplanina; UCI: Unidad de Cuidados Intensivos; VAN: vancomicina. 
TABLA 5. Forma de utilización de cada uno de los antibióticos en estudio empleados para el tratamiento de infecciones

\begin{tabular}{|c|c|c|c|c|}
\hline Formas de utilización & $\begin{array}{c}\text { Total } \\
\mathrm{n}=793\end{array}$ & $\begin{array}{c}\text { VAN } \\
\mathrm{n}=405\end{array}$ & $\begin{array}{c}\text { TPN } \\
\mathrm{n}=293\end{array}$ & $\begin{array}{c}\text { LZD } \\
\mathrm{n}=95\end{array}$ \\
\hline Empírica & $522(65,8)$ & $265(65,4)$ & $199(67,9)$ & $58(61,1)$ \\
\hline No se realizó cultivo & $10(1,9)$ & $5(1,9)$ & $5(2,5)$ & $0(0,0)$ \\
\hline Cultivos negativos (tratamiento empírico) & $217(41,6)$ & $104(39,2)$ & $81(40,7)$ & $32(55,2)$ \\
\hline Microorganismos no CGP & $43(8,2)$ & $21(7,9)$ & $14(7,0)$ & $8(13,8)$ \\
\hline Confirmación de infección por CGP & $252(48,3)$ & $135(50,9)$ & $99(49,7)$ & $18(31,0)$ \\
\hline Específica & $271(34,2)$ & $140(34,6)$ & $94(32,1)$ & $37(38,9)$ \\
\hline Monoterapia & $132(16,0)$ & $73(17,0)$ & $38(12,7)$ & $21(21,9)$ \\
\hline Terapia combinada & $694(84,0)$ & $357(83,0)$ & $262(87,3)$ & $75(78,1)$ \\
\hline Modificación del tratamiento inicial & $224(28,2)$ & $143(35,3)^{*}$ & $67(22,9)^{*}$ & $14(14,7)^{*} \mathrm{p}<0,001$ \\
\hline \multicolumn{5}{|l|}{ Motivo del cambio del tratamiento inicial } \\
\hline Fracaso clínico & $59(26,3)$ & $26(18,2)^{*}$ & $32(47,8)^{*}$ & $1(7,1) p=0,001$ \\
\hline Desescalamiento terapéutico & $128(57,1)$ & $90(62,9)$ & $28(41,8)$ & $10(71,4)$ \\
\hline Insuficiencia renal & $16(7,1)$ & $13(9,1)$ & $3(4,5)$ & $0(0,0)$ \\
\hline Otros motivos & $21(9,4)$ & $14(9,8)$ & $4(5,9)$ & $3(21,5)$ \\
\hline \multicolumn{5}{|l|}{ ATB utilizado en el tratamiento de rescate } \\
\hline VAN & 13 & 0 & 11 & 2 \\
\hline TPN & 13 & 13 & 0 & 0 \\
\hline LZD & 43 & 25 & 18 & 0 \\
\hline Total & 69 & 38 & 29 & 2 \\
\hline
\end{tabular}

Residuos tipificados corregidos con valor absoluto $>1,96$

ATB: antibióticos; CGP: cocos grampositivos; LZD: linezolid; TPN: teicoplanina; VAN: vancomicina.

modificó el tratamiento inicial en 224 casos, siendo más frecuente en los pacientes tratados con VAN. El motivo de cambio predominante fue por el ajuste o desescalamiento terapéutico. Se modificó el tratamiento inicial por fracaso clínico en 59 ocasiones, circunstancia que fue más frecuente en el grupo tratado con TPN y menos habitual en los tratados con LZD. Por otro lado, este fármaco fue el más utilizado en los tratamientos de rescate $(43 / 69,62,3 \%)$.

En total se han identificado 523 CGP tratados con los ATB estudiados, de los que el $43,4 \%$ eran CGP$\mathrm{MR}$, tal como se muestra en la tabla 6 . Han predominado los SARM, S. epidermidis y SCN. La proporción de casos de infección por SARM fue superior en el grupo de LZD (32,7\% frente a 20,4 y 22,3\% respectivamente para VAN y TPN). No se ha registrado aparición de resistencias durante el tratamiento con estos ATB.
Se han identificado efectos adversos probable o posiblemente relacionados con los fármacos estudiados en 36/826 (4,4\%) casos (tabla 7). Estos fueron más frecuentes entre los pacientes tratados con VAN ( $\mathrm{p}=$ $0,031)$, destacando la insuficiencia renal aguda, que se registró en 18 casos de un total de $20(\mathrm{p}=0,003)$ de los que 8 evolucionaron de forma desfavorable. Los casos de trombopenia relacionada con LZD (4/5 casos; $p<0,001)$ tuvieron una evolución favorable. La trombopenia relacionada con VAN evolucionó desfavorablemente; sólo hubo un caso de eritrodermia (síndrome del hombre rojo) tratado con VAN.

\section{DISCUSIÓN}

La principal aportación de este estudio ha sido describir los motivos por los que se emplean los ATB po-

TABLA 6. Distribución de los cocos grampositivos aislados en infecciones de acuerdo con el antibiótico utilizado en su tratamiento

\begin{tabular}{|c|c|c|c|c|}
\hline & $\begin{array}{c}\text { Total } \\
\mathrm{n}=793\end{array}$ & $\begin{array}{c}\text { VAN } \\
\mathrm{n}=405\end{array}$ & $\begin{array}{c}\text { TPN } \\
\mathrm{n}=293\end{array}$ & $\begin{array}{c}\text { LZD } \\
\mathrm{n}=95\end{array}$ \\
\hline Cocos grampositivos & $523(65,9)$ & $275(67,9)$ & $193(65,9)$ & $55(57,8)$ \\
\hline Enterococcus spp. & $9(1,7)$ & $4(1,5)$ & $4(2,1)$ & $1(1,8)$ \\
\hline Enterococcus faecalis & $30(5,7)$ & $16(5,8)$ & $9(4,7)$ & $5(9,1)$ \\
\hline Enterococcus faecium & $19(3,6)$ & $7(2,5)$ & $9(4,7)$ & $3(5,5)$ \\
\hline Staphylococcus aureus MS & $84(16,1)$ & $46(16,7)$ & $31(16,1)$ & $7(12,7)$ \\
\hline Staphylococcus aureus MR & $117(22,4)$ & $56(20,4)$ & $43(22,3)$ & $18(32,7)$ \\
\hline Staphylococcus epidermidis & $100(19,1)$ & $59(21,5)$ & $30(15,5)$ & $11(20,0)$ \\
\hline $\mathrm{SCN}$ & $92(17,6)$ & $40(14,5)$ & $47(24,4)$ & $5(9,1)$ \\
\hline Otros Staphylococcus & $29(5,5)$ & $14(5,1)$ & $13(6,7)$ & $2(3,6)$ \\
\hline Streptococcus pneumoniae & $30(5,7)$ & $22(8,0)$ & $6(3,1)$ & $2(3,6)$ \\
\hline Streptococcus pyogenes & $4(0,8)$ & $3(1,1)$ & $0(0,0)$ & $1(1,8)$ \\
\hline Streptococcus viridans & $4(0,8)$ & $3(1,1)$ & $1(0,5)$ & $0(0,0)$ \\
\hline Streptococcus spp. & $5(1,0)$ & $5(1,8)$ & $0(0,0)$ & $0(0,0)$ \\
\hline CGP-MR & $227(43,4)$ & $105(38,2)$ & $73(37,8)$ & $29(52,7)$ \\
\hline
\end{tabular}

CGP-MR: cocos grampositivos multirresistentes; LZD: linezolid; MR: meticilín-resistente; MS: meticilín-sensible; SCN: Staphylococcus coagulasa negativo; TPN: teicoplanina; VAN: vancomicina. 
TABLA 7. Efectos adversos relacionados probable o posiblemente con los antibióticos en estudio

\begin{tabular}{|c|c|c|c|c|c|}
\hline & $\begin{array}{c}\text { Total } \\
\mathrm{n}=826\end{array}$ & $\begin{array}{c}\text { VAN } \\
\mathrm{n}=430\end{array}$ & $\begin{array}{c}\text { TPN } \\
\mathrm{n}=300\end{array}$ & $\begin{array}{c}\text { LZD } \\
\mathrm{n}=96\end{array}$ & $\mathrm{p}$ \\
\hline Efectos adversos & $36(4,4)$ & $26(6,0)$ & $6(2,0)$ & $4(4,2)$ & \\
\hline Insuficiencia renal & $20(55,6)$ & $18(69,2)^{*}$ & $2(33,3)$ & $0(0,0) *$ & $<0,001$ \\
\hline Rash cutáneo & $6(16,7)$ & $5(19,2)$ & $1(16,7)$ & $0(0,0)$ & \\
\hline Trombopenia & $5(13,9)$ & $1(3,8)^{*}$ & $0(0,0)$ & $4(100)^{*}$ & $<0,001$ \\
\hline Elevación de transaminasas & $1(2,8)$ & $0(0,0)$ & $1(16,7)$ & $0(0,0)$ & \\
\hline Eritema facial & $1(2,8)$ & $0(0,0)$ & $1(16,7)$ & $0(0,0)$ & \\
\hline Síndrome del hombre rojo & $1(2,8)$ & $1(3,8)$ & $0(0,0)$ & $0(0,0)$ & \\
\hline Otros & $2(5,6)$ & $1(3,8)$ & $1(16,7)$ & $0(0,0)$ & \\
\hline
\end{tabular}

Residuos tipificados corregidos con valor absoluto $>1,96$

LZD: linezolid; TPN: teicoplanina; VAN: vancomicina.

tencialmente activos frente a CGP-MR. Se utilizan para el tratamiento de infecciones, mayoritariamente nosocomiales, de adquisición intra-UCI, en especial NVM y BRC. Es de destacar la utilización de todos ellos preferentemente de forma empírica y en combinación con otros ATB, siendo VAN el más utilizado.

La incidencia de infecciones tanto comunitarias como nosocomiales puede llegar a afectar al 40-45\% de los pacientes ingresados en la $\mathrm{UCI}^{5}$. Según datos del ENVIN ${ }^{12}$ más de la mitad de ellos recibe uno o más ATB durante su estancia en la UCI, porcentajes que son coincidentes con otros estudios europeos ${ }^{13}$. El mayor protagonismo de los CGP y el incremento de las cepas multirresistentes ha provocado un notable aumento en la utilización de glucopéptidos, especialmente VAN, en el ámbito hospitalario y también en la UCI. En la última década VAN fue uno de los ATB más utilizados para el tratamiento dirigido de infecciones adquiridas en la UCI. TPN se utiliza menos que VAN, aunque se encuentra entre los 10 antimicrobianos más prescritos en la UCI en infecciones hospitalarias, mientras que el uso de LZD ha aumentado progresivamente desde su incorporación a la farmacopea española a finales de 2001. La utilización de Q/D es prácticamente nula.

Los resultados de nuestro estudio, en el que se recogieron datos de pacientes ingresados en UCI en los años 2003-2004 de una amplia variedad de hospitales de la geografía española, corroboran los porcentajes de utilización descritos. Según los datos del ENVIN del año $2006^{6}$ estos porcentajes han sufrido algunos cambios. Mientras que VAN continúa siendo el principal ATB utilizado (54,2\%), LZD ha alcanzado a TPN (22,8\% para ambos ATB).

Las características de los pacientes en los que se han indicado cada uno de los ATB controlados son diferentes entre sí. En nuestra muestra los pacientes tratados con TPN o LZD estaban más graves al ingreso que los tratados con VAN. Así mismo, hay una mayor proporción de pacientes con sepsis grave/ shock séptico entre los tratados con TPN o LZD que entre los que seguían tratamiento con $\operatorname{VAN}(\mathrm{p}=$ $0,003)$. Estas diferencias sugieren la elección de estos fármacos en situaciones de mayor disfunción orgánica, o en relación con su menor nefrotoxicidad. En el grupo de enfermos tratados con LZD había más pacientes con diabetes mellitus, insuficiencia renal cró- nica, trasplantados de órganos y en tratamiento con corticoides que en los tratados con VAN. Con respecto al grupo tratado con TPN las diferencias con VAN son menores y no significativas, lo que sugiere que LZD está sustituyendo a TPN en el tratamiento de enfermos con insuficiencia renal y/o alteración de las defensas inmunológicas.

La mortalidad ha sido significativamente menor en el grupo de pacientes tratados con VAN, en comparación con los que seguían tratamiento con TPN o LZD ( $p<0,001)$. Esta circunstancia es esperable, teniendo en cuenta que el grupo tratado con VAN tenía menos comorbilidades, menor gravedad al ingreso así como en la forma de presentación de la infección y mejor estado inmunológico.

Los ATB activos frente a CGP-MR se han indicado fundamentalmente para el tratamiento de infecciones hospitalarias, en su mayoría infecciones adquiridas en la UCI. Las indicaciones más frecuentes fueron para tratar NVM y BRC, que en conjunto representan más del $40 \%$ de los tratamientos. Mientras que los glucopéptidos se han empleado preferentemente en el tratamiento de las BRC, LZD se ha utilizado para el tratamiento de NVM. Las indicaciones para la profilaxis han sido muy escasas, destacando en nuestra muestra la de la cirugía cardiaca. En conjunto la utilización de dichos ATB sigue las recomendaciones publicadas para el tratamiento de infecciones producidas por CGP-MR.

La mayoría de los ATB que se emplean para el tratamiento de infecciones en pacientes críticos se inician de forma empírica. En el ENVIN sólo el 25\% de las indicaciones de ATB son dirigidas (con conocimiento del patógeno responsable de la infección). En nuestro estudio los ATB utilizados para el tratamiento de infecciones producidas por CGP-MR fueron dirigidos en el 34,2\% de los casos. Si a ello se añade que en casi la mitad de los tratamientos empíricos se confirmó la etiología de la infección, se puede afirmar que en esta muestra los tratamientos se realizaron con seguridad diagnóstica en el $66 \%$ de las infecciones, porcentaje muy importante teniendo en cuenta que se trata de enfermos críticos en los que el diagnóstico etiológico muchas veces es difícil. El $S$. aureus fue el patógeno que en más ocasiones se identificó, en especial los que eran resistentes a meticilina, lo que se justifica debido a que un grupo impor- 
tante de tratamientos se realizó en pacientes con infecciones pulmonares en su mayoría adquiridas en la UCI, y que el tratamiento se hizo dirigido o con sospecha de infección por CGP-MR. Es de esperar que en el futuro aumenten los tratamientos dirigidos de estas infecciones, dada la posibilidad de llegar a una identificación rápida del SARM mediante la aplicación de técnicas de reacción en cadena de la polime$\operatorname{rasa}^{21,22}$.

El tratamiento inicial se modificó en más de la cuarta parte de los casos, y en su mayoría fue por desescalamiento o ajuste terapéutico $(57,1 \%)$ en función de los resultados microbiológicos, lo que indica una utilización racional de estos ATB. Un 26,3\% de todos los cambios en el tratamiento inicial fue por fracaso clínico, del que casi la mitad estaban tratados con TPN. Esto podría ser debido a que este grupo de pacientes estaba más grave, aunque es posible que la dosis media de $400 \mathrm{mg}$ /día fuera insuficiente, como opinan diversos autores ${ }^{23}$. De los tres ATB estudiados LZD fue el que en menos ocasiones requirió de cambio terapéutico y el más utilizado en los tratamientos de rescate.

En general, la incidencia de efectos adversos ha sido baja, dato que coincide con los de múltiples estudios de estos ATB ${ }^{15-19}$, aunque ha sido mayor en el grupo de VAN. El más destacable por su frecuencia e importancia ha sido la toxicidad renal. Hay que destacar que 18 de los 20 casos registrados estaban tratados con VAN ( $p=0,003)$. No se dispone de información sobre monitorización de niveles plasmáticos de VAN, y es posible que con la dosis media de $2 \mathrm{~g} /$ día utilizada mayoritariamente no se alcancen las concentraciones plasmáticas recomendadas en la actualidad para el tratamiento de infecciones graves ${ }^{24-28}$. Los pocos casos de trombopenia detectados se han relacionado en su mayoría con la utilización de LZD.

En cuanto a las limitaciones de este trabajo, y considerando que se trata de un estudio descriptivo que acota de alguna manera las conclusiones que de él se pueden derivar, tiene la virtud de contar con una muestra amplia y obtenida de un importante número de centros hospitalarios de la geografía española. Por dicho motivo estos resultados no se pueden extrapolar automáticamente a otros países, sobre todo a aquellos en los que la organización socio-sanitaria sea distinta. También hay que tener en cuenta un posible sesgo de reclutamiento, al ser una investigación que ha contado con el apoyo técnico de la industria farmacéutica. Sobre este punto hay que destacar que la posible influencia de esta circunstancia no se ve reflejada en el trabajo, en tanto en cuanto los porcentajes de indicaciones encontrados no varían de los obtenidos en estudios de vigilancia epidemiológica serios y ampliamente reconocidos nacional e internacionalmente, como es el caso del ENVIN.

Como conclusión de este estudio se puede afirmar que la elección de los diversos ATB específicos frente a CGP-MR en el ámbito del paciente crítico se realiza atendiendo a las características, gravedad y comorbilidades de los pacientes. En este ámbito las indicaciones más frecuentes fueron para tratar infec- ciones hospitalarias adquiridas en la UCI, fundamentalmente NVM y BRC en las que se ha llegado a un diagnóstico etiológico de seguridad en un porcentaje importante de casos. Los glucopéptidos fueron los fármacos más utilizados, en especial VAN, aunque LZD ha sido el modificado en menos ocasiones y el más utilizado como tratamiento de rescate. El conjunto de la información indica un empleo racional de estos ATB en el área de atención de pacientes críticos.

\section{Declaración de conflicto de intereses}

Este estudio ha sido patrocinado por el Grupo de Trabajo de Enfermedades Infecciosas de la Sociedad Española de Medicina Intensiva, Crítica y Unidades Coronarias (GTEI-SEMICYUC). Para su desarrollo ha contado con el apoyo técnico de Pfizer. F. Álvarez Lerma ha recibido una beca de Gilead, honorarios por conferencias de Pfizer, Wyeth, Sanofi-Aventis, Gilead, MSD y Novartis y honorarios por asesorías de Pfizer, Novartis, Wyeth y Cephalon. O. Rodríguez Colomo ha recibido apoyo técnico para investigación de Wyeth Farma, honorarios por conferencias de Lilly y Pfizer y honorarios por asesorías de Pfizer.

\section{PROFESIONALES QUE FORMAN EL «GRUPO DE ESTUDIO DE USO DE ANTIBIÓTICOS ACTIVOS FRENTE A COCOS GRAM POSITIVOS MULTIRRESISTENTES EN PACIENTES CRÍTICOS»}

Se describen ordenados en función del número de casos aportados al estudio:

F. Álvarez Lerma e Y. Díaz Buendía. Hospital del Mar. Barcelona (80 casos); B. Álvarez Sánchez. Hospital General Universitario de Alicante (62 casos); R. Oltra Chordá y O. Rodríguez Colomo. Hospital Clínico Universitario. Valencia (51 casos); F. Barcenilla Gaite. Hospital Arnau de Vilanova. Lleida (40 casos); E. Cereijo Martín-Grande. Hospital de La Princesa. Madrid (40 casos); J. Cuñat de la Hoz. Hospital General La Fe. Valencia (40 casos); A. Martínez Pellús. Hospital Virgen de la Arrixaca. Murcia (39 casos); J. Solé Violán y O. Pérez Quevedo. Hospital Dr. Negrín. Las Palmas de Gran Canaria (37 casos); V. González Sanz. Hospital General Miguel Servet. Zaragoza (32 casos); A. Torres Martí. Hospital Clinic i Provincial. Barcelona (32 casos); M. Sánchez García. Hospital Príncipe de Asturias. Alcalá de Henares. Madrid (27 casos); J.M. Flores Cordero y T. Aldabó. Hospital de Traumatología Virgen del Rocío. Sevilla (20 casos); C. León Gil y R. Vicho Pereira. Hospital Nuestra Señora de Valme. Sevilla (20 casos); J.M. Nava Caballero. Hospital Mutua de Terrassa (20 casos); J.C. Pozo Laderas y J.C. Robles Arista. Hospital Reina Sofía. Córdoba (20 casos); M.Á. Arrese Cosculluelas. Hospital Virgen de la Salud. Toledo (19 casos); R. Cabadas. Hospital Policlínico Povisa. Vigo (19 ca- 
sos); F. García López. Hospital General Universitario de Albacete (19 casos); F. García Córdoba. Hospital Morales Meseguer. Murcia (18 casos); M. Robles Marcos y M.M. Latorre López. Hospital Infanta Cristina. Badajoz (18 casos); M.Á. Herranz Casado. Hospital del Río Hortega. Valladolid (16 casos); M.J. Broch Porcar. Hospital de Sagunto. Valencia (15 casos); G. Masdeu Eixarch. Hospital Verge de la Cinta. Tortosa. Tarragona (14 casos); M.Á. Blasco Navalpotro. Hospital Doctor Peset. Valencia (13 casos); A. Rovira Plarromaní. Hospital de la Creu Roja. L'Hospitalet de Llobregat. Barcelona (13 casos); H. Martínez López. Hospital de la Cruz Roja. Córdoba (12 casos); P. Olaechea Astigarraga. Hospital de Galdakao. Galdácano. Vizcaya (12 casos); D. Bravo Sánchez. Hospital Marqués de Valdecilla. Santander (11 casos); A. González Sánchez. Hospital Nuestra Señora del Rosell. Cartagena. Murcia (11 casos); J. Blanco Pérez. Hospital Xeral de Lugo (10 casos); J. Costa Terradas. Hospital de Barcelona (SCIAS) (10 casos); F. Fernández Dorado. Hospital Centro Médico Delfos. Barcelona (10 casos); F. González. Hospital San Cecilio. Granada (10 casos); J. Córdoba Escames. Hospital Comarcal La Inmaculada. Almería (9 casos); J.L. Pérez Vela. Hospital 12 de Octubre. Madrid (8 casos); M. Casanovas Taltavull. Hospital Comarcal de Igualada. Barcelona (6 casos); A. Díaz Lamas. Complejo Hospitalario de Ourense (5 casos); C. Palazón Sánchez. Hospital General Universitario. Murcia (5 casos).

\section{BIBLIOGRAFÍA}

1. Asensio A, Cantón R, Vaqué J, Roselló J, Arribas JL. [Antimicrobial use in Spanish hospitals (EPINE, 1990-1999)]. Med Clin (Barc). 2002;118:730-6.

2. Álvarez-Lerma F, de la Cal MA, Insausti J, Palomar M, Olaechea P and the Spanish Study Group of Surveillance of UCIAcquired Infection. Surveillance of UCI-acquired infection: a Spanish nationwide study. Intensive Care Med. 1996; Suppl 3:317.

3. Valles J, León C, Álvarez Lerma F. Nosocomial bacteremia in critically ill patients: a multicenter study evaluating epidemiology and prognosis. Spanish Collaborative Group for Infections in Intensive Care Units of Sociedad Española de Medicina Intensiva y Unidades Coronarias (SEMIUC). Clin Infect Dis. 1997;24:38795.

4. Álvarez Lerma F, Palomar Martínez M, Olaechea Astigarraga P, Insausti Ordeñana J, Bermejo Fraile B, Cerdá Cerdá E. Estudio nacional de vigilancia de infección nosocomial en Unidades de Cuidados Intensivos. Informe del año 2002. Med Intensiva. 2005;29:1-12.

5. Álvarez-Lerma F, Palomar M, Olaechea P, Otal JJ, Insausti J, Cerdá E y Grupo de Estudio de Vigilancia de Infección Nosocomial en UCI. Estudio Nacional de Vigilancia de Infección Nosocomial en Unidades de Cuidados Intensivos. Informe evolutivo de los años 2003-2005. Med Intensiva. 2007;31:6-17.

6. Grupo de Trabajo de Enfermedades Infecciosas de la Sociedad Española de Medicina Intensiva Crítica y Unidades Coronarias. Estudio Nacional de Vigilancia de Infección Nosocomial en Servicios de Medicina Intensiva (ENVIN-UCI). Informe 2006; SEMICYUC.

7. Richards MJ, Edwards JR, Culver DH, Gaynes RP Nosocomial infections in medical intensive care units in the United States. National Nosocomial Infections Surveillance System. Crit Care Med. 1999;27:853-4.

8. Cardo D, Horan T, Andrus M, Dembinski M, Edwards J, Peavy G, et al. National Nosocomial Infections Surveillance
(NNIS) System Report, data summary from January 1992 through June 2004, issued October 2004. Am J Infect Control. 2004;32: 470-85.

9. Picazo JJ, Betriu C, Rodríguez-Avial I, Culebras E, Gómez M, López F. Grupo VIRA. Vigilancia de resistencias a los antimicrobianos: estudio VIRA 2006. Enferm Infecc Microbiol Clin. 2006;24:617-28.

10. Jones ME, Draghi DC, Thornsberry C, Karlowsky JA, Sahm DF, Wenzel RP. Emerging resistance among bacterial pathogens in the intensive care unit-a European and North American Surveillance study (2000-2002). Ann Clin Microbiol Antimicrob. 2004;3:14.

11. Álvarez Lerma F, Palomar $M$, Insausti J, Olaechea $P$, Cerdá E, Sánchez Godoy J, et al; Grupo de Estudio Nacional de Vigilancia de Infección Nosocomial en UCI. [Staphylococcus aureus nosocomial infections in critically ill patients admitted in intensive care units]. Med Clin (Barc). 2006;126:641-6.

12. Insausti J. Consumo de antibióticos en medicina intensiva. En: Estudio Nacional de Vigilancia de Infección Nosocomial en UCI (ENVIN-UCI). Madrid: Jarpyo editores, S.A.; 2002. p. 129-48.

13. Malacarne P, Rossi C, Bertolini G. GiViTI Group. Antibiotic usage in intensive care units: a pharmaco-epidemiological multicentre study. J Antimicrob Chemother. 2004;54:221-4.

14. Grau S, Álvarez-Lerma F, Marín M, Luis Jimeno J. [Problems and current solutions for the treatment of infections caused by gram-positive microorganisms]. Enferm Infecc Microbiol Clin. 2001;19:393-8.

15. Pigrau C. Oxazolidinonas y glucopéptidos. Enferm Infecc Microbiol Clin. 2003;21:157-65.

16. Ziglam HM, Finch RG. Limitations of presently available glycopeptides in the treatment of Gram-positive infection. Clin Microbiol Infect. 2001;7 Suppl 4:53-65.

17. Goetz MB, Sayers J. Nephrotoxicity of vancomycin and aminoglycoside therapy separately and in combination. J Antimicrob Chemother. 1993;32:325-34.

18. Marchese A, Schito GC. The oxazolidinones as a new family of antimicrobial agent. Clin Microbiol Infect. 2001;7 Suppl 4:66-74.

19. Bouza E, Muñoz P. Linezolid: pharmacokinetic characteristics and clinical studies. Clin Microbiol Infect. 2001;7 Suppl 4:75-82.

20. Bone RC, Balk RA, Cerra FB, Dellinger RP, Fein AM, Knaus WA, et al. Definitions for sepsis and organ failure and guidelines for the use of innovative therapies in sepsis. The ACCP/ SCCM Consensus Conference Committee. American College of Chest Physicians/Society of Critical Care Medicine. Chest. 1992; 101:1644-55.

21. Paule SM, Pasquariello AC, Thomson RB Jr, Kaul KL, Peterson LR. Real-time PCR can rapidly detect methicillin-susceptible and methicillin-resistant Staphylococcus aureus directly from positive blood culture bottles. Am J Clin Pathol. 2005;124: 404-7.

22. Stratidis J, Bia FJ, Edberg SC. Use of real-time polymerase chain reaction for identification of methicillin-resistant Staphylococcus aureus directly from positive blood culture bottles. Diagn Microbiol Infect Dis. 2007;58:199-202.

23. Wilson APR, Grüneberg RN, Neu H. A critical review of the dosage of teicoplanin in Europe and the USA. Int J Antimicrob Agents. 1994;4 Suppl 1:S1-S30.

24. Moise-Broder PA, Forrest A, Birmingham MG, Schentag JJ. Pharmacodynamics of vancomycin and other antimicrobials in patients with Staphylococcus aureus lower respiratory tract infections. Clin Pharmacokinet. 2004;43:925-42.

25. Cruciani M, Gatti G, Lazzarini L, Furlan G, Broccali G, Malena M, et al. Penetration of vancomycin into human lung tissue. J Antimicrob Chemother. 1996;38:865-9.

26. Bingen E, Mariani-Kurkdjian P, Nebbad B. Optimal vancomycin serum level in Staphylococcus aureus infections? Med Mal Infect. 2006;36:439-42.

27. Stevens DL. The role of vancomycin in the treatment paradigm. CID. 2006;42 Suppl 1:42-7.

28. Pea F, Viale P. The antimicrobial therapy puzzle: could pharmacokinetic-pharmacodynamic relationships be helpful in addressing the issue of appropriate pneumonia treatment in critically ill patients? CID. 2006;42:1764-71. 